LECT URES

\author{
ON THE
}

\section{DIAGNOSIS AND SURGICAL TREATMENT OF ABDOMINAL TUMOURS.}

\section{Delivered at the Royal College of Surgeons of England.}

BY

T. S P E N C E R W E L L S, F.R.C.S.,

Professor of Surgery and Pathology in the College; and Consulting Surgeon to the Samaritan Hospital.

LECTURE II-Fune $12 t h, 1878$.

Semisolid Abdominal Tumours; Different Kinds of Ovarian Tumours - their Diagnosis-Complications with Pregnancy; Extra-uterine Presnancy-Specimens illustrating various other Conditions resembling Ovarian Tumours; Fibroid and Fibro-cystic Uterine Tumours; Tiumours of Abdominal Wall ; Tumours of Omentum and Mesentery, of Liver, Spleen, Kidney, and Mesenteric Glands; Cancer and Tubercle; Aneurism; Hamatocele and Pelvic Abscess; Facal Accumulation; Phantom Tumours.

A $\mathrm{r}$ the lecture on Monday, I was speaking about the chemical qualities of the fluid removed in tapping ovarian cysts; and yesterday, I had an opportunity of removing from a young lady sixteen pints of fluid, of which this is a portion. You will see that it is very nearly of the colour of distilled water. Through this green bottle, there is a little tint visible ; but if it be put into a white glass jar, there is no colour to be seen, only the very slightest opalescence. The specific gravity is 1006, which is a little more than we occasionally find in these fluids removed from simple cysts. Then the albumen which it contains is not true albumen, but the form I have described to you as being the coagulum which, formed by heat, is redissolved upon the addition of boiling acetic acid; so that the albumen is not true albumen but paralbumen. On examining the deposit, there is nothing whatever to be seen, and the fluid may be looked upon as a very fair or typical specimen of that from a simple or single ovarian cyst, or of an extra-ovarian cyst-a cyst formed in the broad ligament near one or other ovary. Yesterday, also, I had the opportunity of removing a single ovarian cyst ; and thinking it might interest you to see it, I have brought it here. You will see what a very thin-walled cyst it is, completely single, absolutely free from all adhesions, and the only opening in it is that made by the trocar in emptying the fluid from it, in order that the empty cyst might follow the fluid removed. The case very well bears out what I was saying about the difficulty occasionally met with in ascertaining whether the fluid in a large cyst is really in a cyst or whether it is free in the peritoneal cavity. The lady was fifty-seven years of age. Last summer, the abdomen began to increase in size. She consulted two or three physicians, who all told her she had liverdisease and dropsy, but whether the dropsy depended on cancer or tubercle, they did not seem very well to know. Then Dr. Risdon Bennett, the President of the College of Physicians, saw her. He had no doubt at all about the fluid being encysted, and advised the patient to see me. I at once advised that she should be tapped, seeing that the cyst was a single cyst, and possibly it might be one of those cases in which the fluid would not re-form after tapping. I tapped it last October, and eighteen pints of fluid were removed. The lady improved very much in health, and went to Brighton. Last March, it was again tapped, about the same quantity of fluid being removed. It is a curious instance of the different reports one gets about the quantity of fluid removed. The surgeon who tapped her wrote to me that he removed "seven gallons of fluid". I thought it very strange, and on questioning the patient, I found instead of being seven gallons it was seven quarts. That is the kind of mistake one sometimes meets with inadvertently made in the reports of cases. After the second tapping, the cyst refilled; and as it seemed a case in which mere tapping would be of very little use, it was arranged I should remove the cyst yesterday, which I did, and here it is. It is a thin transparent cyst, which yesterday contained eighteen pints of fluid, and which came out through a very small opening as soon as it was emptied. As the fluid escaped, so the empty cyst came out. There is nothing more to be said, except that the patient is perfectly free from fever, and in all probability will do very well.
Then I was going on, at the close of the last lecture, to speak about the microscopical examination of the fluids removed by tapping, and I was about to show you some of these diagrams, which show the last additions to our knowledge on this question. Long ago, Dr. Hughes Bennett of Edinburgh described certain cells, which are extremely well figured in his little book-cells which he considered to be quite distinctive of the fluid removed from ovarian cysts. He believed that when those cells were found in fluid, it could come only from an ovarian cyst, and from nothing else. This examination was afterwards carried on, and in my own book on Diseases of the Ovaries, which I formerly mentioned to you, there are about one hundred figures, em. bracing nearly everything which had been described up to that time as having been found in cysts of various kinds. Mr. Nunn had previously described some of the same sort of granular cells or epithelial cells and granules ; and then, in America, Dr. Drysdale, the son-in-law of Dr. Atlee of Philadelphia, having the opportunity of examining nearly all the cysts removed by Dr. Atlee, carried on this investigation, and described, as "ovarian granule-cells", the cells which are pretty well shown on this diagram-that sort of cell which differs very little from the cell which Dr. Bennett described years before as characteristic of the fluid of ovarian dropsy. I have also drawn here some ordinary red blood-corpuscles, in order that you might make the comparison of the size in which these things appear as compared with a bloodcorpuscle under the field of the microscope. (Figs. I and 2.) We

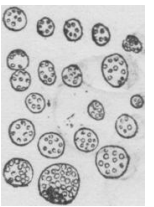

Fig. r.

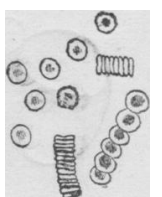

Fig. 2. suppose these to be simply the nuclei of the epithelial cells which line the interior of the cyst. The scales are thrown off, the cell-wall breaks down, and the nucleus remains.

After a good deal of controversy as to the exact meaning and value of these things, whether they are found in ovarian cysts only, or in the kidney, or the spleen, Mr. Knowsley Thornton, who for some time past has examined a great many of the tumours which I have removed, made a great addition to our knowledge in pointing out that, in addition to these cells of Drysdale, which are common only in simple or innocent ovarian tumours, in malignant tumours you have these very characteristic groups of cells of different sizes. He describes them as large numbers of characteristic groups of large pear-shaped, round, or oval cells, containing a granular material with one or several large clear nuclei, with nucleoli and a number of transparent globules or vacuoles. The cells composing the groups are many of them very large, but the great variety in size and shape is the marked feature of the group. If you: will bear these different forms in mind, and these different cells, I believe you will find that they are characteristic, and of great value in the examination of these fluids, putting us on our guard when we have to deal with tumours doubtfully malignant. If these large groups of cells be seen, one may be pretty certain the tumour is malignant of sorne kind; or if they be found in fluid removed from the peritoneal cavity, probably a sort of infecting process has been going on in the peritoneum from the rupture of an ovarian cyst of a malignant character; these cells may have planted themselves upon some part of the peritoneum and multiplied.
Fig. 3.

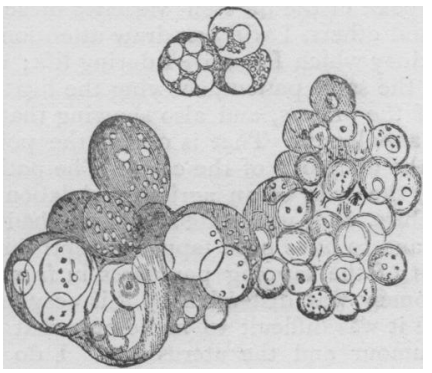

Fig. 4

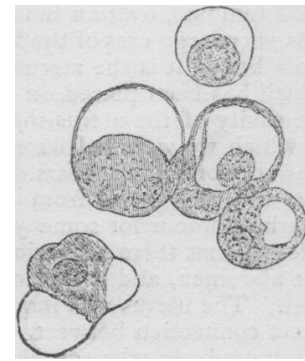

Fig. 5
Fig. 3 is a group of vacuolating cells from ovarian fluid (rare). Fig. 4 is a group of cells from peritoneal fluid in a case of cancer 
the pancreas, omentum, etc., freshly drawn and no reagents used. In fig. 5 are single cells and groups from the sediment of peritoneal fluid treated with nitric acid and logwood. The single cells show the vacuole substance (colloid) pressed to the outer surface by the action of the reagents. The centre cell in the group shows the colloid substance shrivelled from the same cause. The case was one of slowgrowing sarcoma of the ovary. Figs. 6, 7, and 8 represent cell-groups

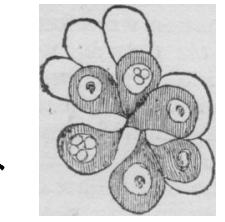

Fig. 6.

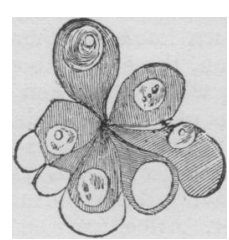

Fig. 7.

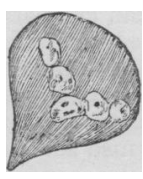

Fig. 8. and a single cell from the same fluid as No. 4, but treated with nitrate of silver. There are vacuoles both in the cells and nuclei; in the cells pressed to the circumference by the action of the reagent. The single cell has the characteristic balloon shape, and shows a curious row of nuclei. Fig. 9 gives a view of a group of cells from peritoneal

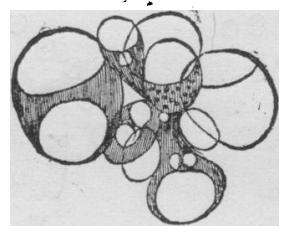

Fig. 9.

fluid (fresh, no reagent used) in a case of rapidly growing sarcoma of ovaries and uterus. The nuclei have all disappeared, and the cells are almost destroyed by vacuolation. All these drawings were made for me by Mr. Thornton himself.

This last drawing (fig. Io) shows Dr. Drysdale's sketch of the fluid

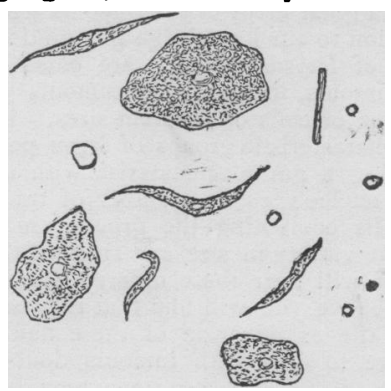

Fig. 10.

removed in a case of fibro-cystic tumour of the uterus, with what he calls "fibre-cells". I need not say more now about the microscopical appearances found in these fluids. The work of Dr. Atlee, with Dr. Drysdale's own illustrations, is very well known, and may be found in the library.

I think, before passing on to speak of the different varieties of semisolid tumours, ovarian tumours and others, I will just draw attention to this very large cyst of the left kidney which I removed during life; and close beside it is the uterus from the same patient, showing the ligature which has been placed on one of the ureters, and also showing that in the cavity of the uterus there is a polypus. That is one of the points in which we were led wrong in the diagnosis of the case. The patient was a woman 29 years of age, the wife of an agricultural labourer. She was sent to me from Yorkshire, and was stated to have had an ovarian tumour for some years, and to have been tapped several times. I found that there were cicatrices of the tapping punctures in front of the abdomen, and that the abdomen was distended by this very large cyst. The uterus was large, and it was difficult to make out what the exact connection between the tumour and the uterus was. I do not think anybody who saw the case had any doubt that it was an ovarian cyst; the only question in the case was whether or not the woman was pregnant, and I proceeded to the operation without the faintest shadow of a doubt that I was going to remove an ovarian cyst. But, as soon as the abdominal wall was divided, I found I was coming upon something peculiar. I could not find the peritoneum as one generally does, but came rather upon the cyst-wall covered with a loose layer of cellular tissue, and I then began to think I was dealing with an extra ovarian cyst, the sort of cyst one often finds covered by an expansion of one or other of the broad ligaments ; so I emptied the cyst, expecting to be able to separate it more easily. When it was empty, I began to try to separate the adhesions. I found the uterus and both ovaries quite healthy, and then, of course, came the question of what should be done. I was disposed to leave it alone, and see what drainage would effect; but so many adhesions had been separated, that that seemed to be rather hazardous; and, although I perceived it was a kidney-cyst, I thought I had better remove it, and so I did. I tied the vessels, and the operation seemed to have been completed satisfactorily. The tumour was removed, and there was no very great loss of blood. The patient was put to bed, and for some time looked as if she were likely to go on pretty well; but, on the fourth day, she died, without any very characteristic symptoms having been observed. There was nothing peculiar in the urine, and nothing more than one commonly sees in cases of ovariotomy where the patient gets some amount of fever after the operation. On examining the tumour, it is seen very distinctly that the kidney has been expanded, as it were, into that large cyst. I thought it might be interesting to bring this specimen under your notice, as showing that we may get very large single cysts simulating ovarian cysts; and that, when they are associated with a large uterus and with a history of ovarian fluid having been removed at previous tappings, it would take a great deal of care indeed to avoid this sort of error.

The case leads me to speak of another which was somewhat similar, in which we did not make a mistake in diagnosis. A certain amount of pointing in the right loin led to a more careful examination of the state of the case, and making an opening into the loin, introducing a drainage-tube, and treating it antiseptically, keeping the end of the tube covered with a sponge wetted with carbolic acid and occasionally injecting, that patient was completely cured. I do not think the tumour was so large as the first, but still it did contain a very large quantity of fluid, and fluid which, singularly enough, had a great deal of cholesterine in it. I do not think this has been previously noticed in renal cysts. I will not say more about renal cysts at present, and will proceed in the order which I have laid down in the printed prospectus of these lectures to speak of the semisolid abdominal tumours which one may meet with in great variety. Of these we have several specimens on the table.

Here are four very good specimens of encysted tumours. I will just say a word about the different kinds of ovarian tumour, passing on from the simple single kind which is there. Here is a very good specimen of the multilocular form, which has been very well injected, showing the arrangement of the vessels of the walls of the cyst; and here is an interesting specimen of $a^{\circ}$ cyst in the ovary from a child only five woeks old. Oddly enough, there were twins; one died at five weeks and one at seven weeks, and they both had ovarian cysts. There was nothing wrong about the mother, who was a healthy woman.

Solid tumours in the ovary are much more rare than semisolid ones, but here is a good specimen of a solid tumour which I removed some years ago from a young lady nineteen or twenty years of age, a patient of Mr. Prescott Hewett-a case of perfectly characteristic soft cancer of the ovary. She went on well at first; but, a day or two after the stitches were removed, there was a partial reopening of the wound, accidentally caused by coughing ; peritonitis followed, and death. I do not suppose the patient would have ultimately recovered after such a character of growth as that; but still there would have been a temporary recovery, had it not been for that accidental occurrence.

The different kinds of ovarian tumours and the diagnosis of adhesion may be made out by some little attention in the examination of a patient. We can ascertain with a little care whether a cyst is a single cyst with limpid contents, or whether the contents are likely to be viscid, and this will assist us in forming some opinion as to whether one should be content with tapping and drainage, or whether it would probably be ultimately necessary to resort to ovariotomy. The reasons which would induce us to suppose that the cyst is single, and that it probably may be cured by tapping, are that it may have existed for many years without any great effect upon the general health of the patient; or, on the other hand, that it has formed with such extreme rapidity that it is almost certainly mistaken for ascites. You see a young woman whose abdomen has rapidly enlarged (one may sometimes see this one's self, but it is more frequently reported) ; but I saw it in the case of a young lady I examined many years ago with Dr. Rigby. Neither Dr. Rigby nor I was at first certain that we could 
discover anything in the abdomen; we thought we could make out a little dulness and doubtful fluctuation, but there was no enlargement, and yet, in three weeks, that cyst was as large as an adult head. It rapidly increased, and had to be removed in three weeks after the first examination. That is a very rare and exceptional case. When you get this very rapid formation of a cyst, it is pretty certain the cyst is a single one ; and so also if the cyst have existed a good many years, and the patient have not suffered much from it, it is probably single; and these are cases in which tapping alone may not only afford temporary relief to the patient, as it does, but may possibly complete the cure the fluid may never re-form. In such a case as this, one can detect no hardening of the cyst wall, no nodules, and the surface is so smooth one can make up one's mind that there are no groups of secondary cysts about it, and that it is practically unilocular. When you get resistance to the wave of fluctuation in different directions, then you may also be pretty certain that there are septa dividing the cyst into different portions, and that it is of a multilocular character, and in that case tapping would be of no use ; although one compartment might be emptied, the others would remain full. Supposing we find cartilaginnus or hard bony projections, or nodules, in different parts of the cyst, then the probability is strong that the tumour is one of the forms of dermoid tumour, in which we may find cartilage, bone, fat, hair, teeth, and other things, of which we have some good specimens in the museum. Occasionally one begins to doubt whether both ovaries are affected or not. We may meet with a sulcus going immediately down the abdomen, and, if we find intestine there, in that case it is extremely probable both ovaries are affected. Sometimes this is deceptive. I have seen a very large sulcus on the surface of one ovarian tumour, made by the Fallopian tube pressing it in the centre, so that there was a very distinct bulge on either side, and we felt almost certain that both ovaries were affected, whereas only one was diseased. The belief of Boinet, that one can foretell before tapping the character and colour and consistence of the fluid, has a certain amount of foundation. The progress of the disease, the more or less acute pain complained of, the other signs of inflammation more or less rapid, and the state of the general health of the patient, will lead to suspicion probably that one has some purulent fluid, or mixture of blood and serum, or pus in a cyst; if there have been a rise of temperature at night, loss of appetite, and other signs of fever, you may be pretty certain there has been some inflammatory change going on in the substance of the tumour or cyst, which will lead to changes in the condition and character of the fluid.

The solid tumours I need not say anything more about, except that they do not fluctuate; they are solid. These are much rarer than cases where one finds a portion of the tumour solid and a portion of it fluctuating.

Then with regard to the diagnosis of adhesions, it used to be thought a matter of very great consequence to determine, before undertaking to remove an ovarian tumour, whether it was adherent or not; and, in the early days of ovariotomy, great trouble used to be taken by Dr. Frederic Bird and others to ascertain, if they could, whether the tumour was free. They would tap and watch carefully the way the cannula moved as the cyst emptied, and Dr. Bird used to put needles through the abdominal wall into the cyst, to see the way in which the ends of the needles were deflected, and, if there were signs that the cyst was adherent, then it was not considered a case in which ovariotomy ough to be attempted. When I commenced practice in this particular department of surgery, I soon began to doubt whether this was a point of much consequence, and, when one began to number cases by hundreds, there seemed to be very little difference in result between cases of ad hesion and of non-adhesion, so that I do not think we need give more time or attention to this point, but it is as well to remind you of it.

I have here some specimens of bone and teeth, from cysts which I removed, prepared by Dr. Junker, who for five years was in Japan teaching the Japanese surgery, establishing a surgical school there, and carrying the benefits of our surgical knowledge to the Japanese. They illustrate extremely well the forms of bone-like matter which one occasionally meets with in dermoid cysts of the ovary.

To revert for a moment to the subject of diagnosis of adhesions : practically, I think, the question of extent of adhesion to the abdominal wall is not of much consequence. The result is pretty much the same, so far as the adhesions go, to the patient; but the amount and intimacy of pelvic adhesions are matters of very much greater moment. Supposing an ovarian cyst is adherent low down in the pelvis, between the uterus and rectum or the uterus and bladder, or on either side, the attempt to separate it is necessarily a dangerous one. If any bloodvessels be torn, it is difficult to find them. Although by artificial means one can occasionally throw a strong ray of light to the bottom of the pelvis and secure a bleeding vessel, yet it is troublesome and difficult to do; and the results of these cases are by no means so satis- factory as when there are only adhesions to the abdominal wall. One can pretty well ascertain if there be such adhesions as these, by a careful examination of the pelvis by the vagina, whether the uterus is fixed to a portion of the tumour. You find that they do not move with the position of the patient; they do not move when she coughs; they do not move when the shoulders are lowered and the hips raised; and it is impossible, either with the uterine sound or in any other way, to separate the uterus from these pelvic portions of the adherent tumours. Occasionally there will be an ovarian tumour low down in the pelvis which does move, from which the uterus can be separated, and need not interfere at all with Qvariotomy ; and in such cases it is sometimes quite curious to hear the rush of air into the bottom of the pelvis when the lower portion of the cyst is separated from the hollow of the sacrum. Air rushes down with a gurgle, the tumour is easily brought up, and there is no more difficulty than in an ordinary case. It occasionally becomes very doubtful whether an ovarian tumour is simply jammed down into the pelvis, or whether it is fixed there by strong adhesion; but still I think this can be made out by care-ascertaining whether the uterus is movable; ascertaining whether the lower portion of the cyst moves in different positions of the patient; and, if it really be firmly fixed, whether the sensation to the finger is that it has been absolutely glued down to the bottom of the pelvis. If there be this intimate adhesion pretty low down, as a rule I think the operation is not one that is likely to succeed.

The specimens on the table illustrate extremely well a number of conditions which may be mistaken for ovarian tumours, and which frequently are mistaken for them. The fibroid and fibro-cystic uterine tumours are those which are most commonly mistaken, and there will be other specimens of this kind brought down at the last lecture; but there is one here which is a specimen of a case of very great interest, in which Mr. Adams removed an extra-uterine fotus from the abdomen. Here is another, in which a fœetus was retained for fifty-two years in the abdomen. I do not suppose either of these cases would have been mistaken for an ovarian tumour; but still, in calculating what one may meet with, it is well to bear in mind that that sort of thing may exist. In the last lecture, we will have other specimens of fibroid and fibro-cystic tumours of the uterus brought down, to show how closely some of them resemble ovarian tumours and what a large size some of them attain. Here is a very beautiful specimen of melanosis of the ovary-a specimen not often seen. Here are tumours of the spleen. This is a spleen which I removed during the life of the patient ; it weighed nine pounds, and the patient lived seven days. The case I need not go into further. In two other cases, I removed the spleen just about the same size ; but in neither of the three did the patient survive. There was only one that died from hæmorrhage; in the other two, there was no hæmorrhage, nor much peritonitis; but there was a quantity of white clot on the right side of the heart, which seemed to be the cause of death. The diagnosis was made out with great ease in those cases. One was a case of Sir William Jenner's, in which he made out disease of the spleen as having existed some long time before. There was some alteration in the white blood-corpuscles; but Sir William Jenner thought it was a case in which one might very fairly and reasonably remove the spleen, and the patient seemed to be extremely likely to recover. She went on well for seven days; and at the end of the seventh day, without any reason that one could see, she died quite suddenly. But the reason was manifest after death : there was a very large clot on the right side of the heart.

Here are numerous cases of cancer in different parts of the abdo. men; and these varieties of malignant disease in the abdomen lead very often to mistakes with regard to ovarian tumours. There have been a great many cases of attempted ovariotomy where no ovarian disease at all has been found, but the tumour has proved to be cancer of some one or other of the organs of the abdomen-the liver, for instance. Here are cases of liver-disease and disease of the omentum which have led to distinct mistakes. Here is a case of disease of a portion of the omentum which was mistaken for pregnancy. After death, it was found out that there was disease of the omentum, of which this is a portion. Here is a case of enlarged liver. Here is a case of dropsy of the gall-bladder; this of course, if larger, would make a considerable tumour; and many of you will, perhaps, read with interest next week the continuation of a paper begun by Dr. Marion Sims last week in the BRITISH MEDICAL JOURNAL, in which he relates how he opened the gall-bladder, sewed the opening to the wound in the abdominal wall, and turned out a very large number of gall-stones. I once had an operation considered in consultation about a patient in Kentish Town who had a very large tumour, and the great probability was that there were gall-stones; but there was also evidence that there was malignant disease of the liver, and nothing was done. I am afraid to say the number of gall-stones that were found after her death, but I 
think one thousand eight hundred; and beside that the gall-bladder was very much thickened by cancer. Here is a specimen of cancer of the liver which would have made a very large tumour; there is one of melanosis of the liver. Here is one of tubercle of the liver-a very rare specimen; and here is one of hydatid of the spleen. These hydatid cysts occasionally lead to mistakes. In my work on Diseases of the Ovaries, there is a picture of a woman taken from a photograph, from whom I removed successfully a very large hydatid cyst which we had correctly diagnosed after tapping. The limpid fluid removed at once attaacted attention. I examined the deposit, and found it characteristic of hydatid disease; so, after a time, as the woman seemed to be likely to die, I removed the hydatid cyst. She completely recovered.

Aneurism occasionally may be met with. Here are one or two good specimens of aneurism of the aorta. It is not likely that aneurism of the abdominal aorta will be mistaken for an ovarian tumour, but still it is well to bear this in mind. I know of one case in which an aneurism of the aorta low down, just where it bifurcates, was tapped through the vagina, believing it to be a pelvic abscess. That lady died upon the table in a very few minutes. So it is just as well to remember that these large aneurisms of the abdominal aorta should be considered in forming one's diagnosis.

With regard to hæmatocele and pelvic abscess, I need not say much here, except to put younger men upon their guard to remember these do form occasionally of so large a size as to be mistaken for ovarian or other tumours. I have seen a hæmatocele extend considerably above the level of the umbilicus, and a pelvic abscess almost as large.

Fæcal accumulation, too, but rarely leads to error. Occasionally one is called in to cases where accumulations of large quantities of fæces do lead to mistake, and the supposed tumour disappears after their removal.

Then, a word as to those resonant enlargements of the abdomen which have been called phantom tumours. There is now in the Samaritan Hospital a young woman with an apparently manifest abdominal tumour. The abdomen was prominent, and felt hard; but, on percussion, was distinctly resonant everywhere. She looked in perfectly good health, and, on putting her under the influence of chloroform, the swelling entirely disappeared. There has been a certain amount of doubt as to the exact pathology of these things, whether it is that the diaphragm is pressed downwards, the liver pressed forwards, and a certain amount of distension of the intestines accompanies the arching forwards of the recti ; but certainly occasionally they do look very much like tumours which disappear entirely when the patient is deeply narcotised. I have seen the same thing in a man. I remember a soldier came down to Smyrna from the Crimea with a tumour which excited a good deal of attention amongst the surgeons at Smyrna, but, on putting the man under the influence of chloroform, it entirely disappeared.

With regard to pregnancy, the ordinary signs of pregnancy, of course, should be borne in mind in any case of abdominal tumour in a woman. Whether the woman is single, or married, or a widow, this complication may arise. I need not enter into the different signs of pregnancy but I wish you to bear in mind that not only may pregnancy be mistaken for ovarian disease, or uterine disease, or the converse, but that in many cases pregnancy does complicate ovarian disease. A woman may have a large ovarian tumour, and then she may become pregnant; and the question of the treatment of a woman who has an ovarian tumour and is also pregnant is one I shall have to speak of when I have said something about ovariotomy.

I do not think there is a specimen here, I doubt whether there is in the Museum, of what is called " movable kidney". I believe there have been only one or two such specimens shown after death at the Pathological Society, but I have occasionally seen tumours in the abdomen which appeared, as far as one could tell, to be instances of movable kidneys, either enlarged or of their natural size. One case was such a remarkable one that I will detain you a moment to tell you ahout it. A lady came to me believing she had, or having been told she had, a movable right kidney. I saw her with Dr. Wilson Fox, and we both agreed that was the nature of the disease. For some years it went on, and I saw her occasionally, under the impression that she was suffering from a movable kidney. Then she became pregnant, and pregnancy went on to its natural termination, and a healthy child was born. Soon afterwards, she began to suffer from an ovarian cyst on the left side, the movable kidney still being on the right side; and the ovarian cyst increased so much that it was decided I should perform ovariotomy, which I did. I said at the time "I will see what that movable kidney is now"; and, after I had removed the ovarian cyst on the left side, I felt what the supposed kidney was, and took it out; and then I found it was the right ovary, but it was attached by a pedicle fully a foot long. It had been held up under the right false ribs by the merest little film of adhesion. Quite a small patch of adhesion kept this right ovary, which was about the size of my fist and very much of the shape of a kidney, just in the position of a movable kidney. The patient recovered, and remained in good health.

Then, as to cancer of the ovary. Here are some specimens. Here is cancer of the ovary; and here is cancer of the kidney. There is a specimen also of a tumour of the abdominal wall. This illustrates a mistake occasionally made in practice : in the treatment of a supposed abdominal tumour, it is found that the tumour is not of the abdomen at all, but is simply a tumour of the abdominal wall. I remember years ago being called to see a patient, who came from Portugal, supposed to have ovarian tumour, and I expressed a strong opinion that it was not ovarian, but simply a tumour of the abdominal wall, and decided that it should be left alone; but Mr. Baker Brown afterwards saw the patient, and removed it. He removed a very large portion of the abdominal wall with it. He removed a bit of the peritoneum as large as the palm of my hand, and yet the patient made a most complete recovery. The tumour was a fibro-plastic tumour of the abdominal wall of a considerable size, and the portion of the abdominal wall removed with it was two or three inches in breadth and three or four in length. Yet the abdominal wall, which was brought together without much tension, healed up, and the woman recovered almost as well as if the operation had been one of the most simple character.

\section{THE POLYURIA OF GRANULAR KIDNEY.}

By GEORGE JOHNSON, M.D., F.R.S.,

Professor of Clinical Medicine; Senior Physician to King's College Hospital; etc.

HAVING neither time nor inclination for a controversy on the above subject, I am nevertheless anxious not to be misunderstood. In my paper in the Journal of May 25th, I expressed my dissent from the theory which assumes that the copious secretion of urine by a granular kidney is the result of increased pressure on the Ma lpighian capillaries consequent on obstruction in the intertubular capillaries. I maintain that this theory is utterly inadequate to explain the phenomena, and that the attempt to give them this merely mechanical explanation tends to mislead and retard the progress of actual knowledge. I believe, too, that the results of the impeded renal circulation consequent on cardiac disease are fairly comparable with those which would follow upon an intertubular capillary obstruction. The results in both cases would be, of course, a general diminution of the blood-current through the kidney, a gradual increase of pressure on the Malpighian capillaries, a scanty secretion of water, and ultimately a more or less copious transudation of albumen into the uriniferous tubes. These are the phenomena which are commonly observed in cases of large Bright's kidney, when the swollen uriniferous tubes compress the intertubular plexus, and thus cause a backward strain upon the Malpighian capillaries.

It is to be borne in mind that there is no relation whatever between increased pressure on the Malpighian capillaries consequent on an obstruction in front and such increase of pressure as may result from relaxation of the afferent arterioles. It is to the latter phenomenon that reference is made by Dr. M. Foster in the chapter of his TextBook of Physiology to which Dr. Saundby directs attention, and this phenomenon is not now in question. While I believe that no trustworthy inference can be drawn from such an experiment as that of Dr. Grützner to which Dr. Saundby refers, I feel called upon to correct the erroneous assumption that the contraction of the hypertrophied muscular arterioles must render the kidney anæmic. Dr. Saundby, in raising this objection, overlooks the fact that the resistance offered by the arterioles is more or less completely counterbalanced by the increased injecting force of the hypertrophied left ventricle. And, again, it is not to be supposed that the renal arterioles are in a constant state of extreme contraction, or that their muscular walls, even in the advanced stages of a granular kidney, are beyond the control of the vaso-motor nervous influence by which circulation and secretion are co-ordinated. We may reasonably suppose that, while the regulating influence of the hypertrophied afferent arterioles prevents undue strain upon the Malpighian capillaries from the injecting force of the strong left ventricle, "there is no shutting off of the blood from the glomeruli", but the blood-supply to the gland is sufficient for the purposes of secretion to any amount. 